\title{
Effect of antioxidants on the egg incubation quality and productivity of chickens
}

\author{
K.S. Ostrenko, V.P. Galochkina
}

All-Russian research Institute of physiology, biochemistry and animal nutrition - branch of the Federal research center of animal husbandry - academician L. K. Ernst, Borovsk, Kaluga region, Russia

E-mail: Ostrenkoks@gmail.com

Journal of Livestock Science (ISSN online 2277-6214) 12: 31-36

Received on 2/9/20; Accepted on 29/11/2020; published on 4/1/2021

doi. 10.33259/JLivestSci.2021.31-36

\begin{abstract}
The main purpose of the study was to study the effectiveness of water-soluble antioxidants on the physiological and zootechni cal indicators of egg incubation and offspring hatchability.The research was carried out on two groups of laying hens of a custom herd, selected by random sampling of one hundred heads per group. For 41 days, the experimental group of chickens received a basic diet enriched with dihydroethoxychine (DGE), in order to increase the antioxidant status at a dosage of $100 \mathrm{mg} / \mathrm{kg}$ of feed. Sampling was performed from the axillary vein on the 25th day of application of the additive $(n=5)$ for physiological and biochemical studies. In the course of the study, it was determined that in the experimental group, the concentration of cholesterol in the fraction of highdensity lipoproteins increased twofold $(\mathrm{P}<0.01)$ and in the fraction of low-density lipoproteins decreased by almost $50 \%(\mathrm{P}<0.01)$ against the control. The concentration of malondialdehyde (MDA) in the blood of chickens in the experimental group was $82.00 \%$ relative to that in the control, and in the yolk of the egg - only $37.42 \%$. Egg production of chickens in the experimental group was higher than in the control by $7.3 \%(\mathrm{P}<0.1)$. Hatchability of chickens in the experimental group increased by $14.6 \%(\mathrm{P}<0.01)$ relative to control. It was concluded that the tested water-soluble antioxidant has a positive effect on the biological functions and productive parameters of laying hens.
\end{abstract}

Keywords: chicken; eggs; antioxidant; dihydroethidium; cholesterol, 
Ostrenko \& Galochkina 2021/ J. Livestock Sci. 12: 31-36

\section{Introduction}

Animals and poultry raised in modern industrial complexes are exposed to numerous technogenic stress factors. This is especially true for the bird. This leads to such undesirable effects as a general deterioration in health, a decrease in resistance to infectious and non-communicable diseases, stress, inadequate response even to minor changes in conditions of detention and adverse environmental influences. As a result, there is a decrease in productivity, cost overruns for the production of products, its cost increase with quality deterioration (Buzlama 1996, Galochkin 2008, Bindoli 2005).

Adaptation capabilities are genetically determined. However, in highly productive animals and birds, typical representative of which are laying hens, these opportunities are significantly reduced as a result of many years of unilaterally directed selection for maximum productivity (hypertrophied egg production) to the detriment of a number of other vital functions.

In this regard, the functional and adaptive capabilities of laying hens to changing environmental conditions have decreased and the protective functions of the body have weakened. In such a situation, the need for the use of drugs of biologically active substances, including antioxidants, is growing (Galochkin et al., 2009, Reyes et al., 2005, Travis. et al., 1989). The problem of the formation of the antioxidant status of the organism of higher animals and birds is given ever-increasing attention. New aspects of the regulatory role and the effect of antioxidants on the metabolic processes in the human, animal and bird organism continue to be revealed, an intensive search is underway for new, more effective antioxidants using methods of chemical synthesis and preparative isolation from natural objects (Lauridsen et al., 1995, Middleton. et al., 2001, Ostrenko. et al., 2018, Leong et al.,1992, Wang et al., 1997). The use of antioxidants in the diet of broiler chicken have extensively documented recently (Yesuf et al, 2017, Sethy et al 2017 and Alimohammadi-Saraei et al 2018).

Earlier, we first studied the effectiveness of the inclusion of a new antioxidant dihydroethoxyquin in the diets of broiler chickens (Bindoli 2005). In contrast to ethoxyquin (Santokhin, 6-ethoxy-2,2,4-trimethyl-1,2dihydroquinoline), which is widely used in feeding birds and animals, dihydroethoxyquin (DHE, 6- ethoxy-2,2,4trimethyl -1,2,3,4 - tetrahydroquinoline) there are two more hydrogen atoms, which gives it a number of new useful qualities. This compound, unlike ethoxyquin, which is a lipophilic liquid, is a solid and water-soluble substance. These properties distinguish it from fat-soluble ethoxyquin. Water- soluble antioxidants are significantly less than fat-soluble antioxidants. DHE is more technologically advanced in the preparation of animal feed and feed mixtures. It can be used not only with food, but also with drinking water. Moreover, its toxicity does not exceed the toxicity of ethoxyquin.

Objective of the study- There is need to develop new, more effective ways of rational feeding of broodstock hens.

\section{Materials and methods}

The experiment was conducted at the Vasilievskaya poultry farm, Penza region, $53^{\circ} 27^{\prime} 23.5^{\prime \prime} \mathrm{N}$ $45^{\circ} 16^{\prime} 10.2^{\prime \prime E}$. Laboratory studies were performed in laboratoru Immunobiotechnology and Microbiology AllRussian research Institute of physiology, biochemistry and animal nutrition - branch of the Federal research center of animal husbandry - academician L. K. Ernst. The poultry farm uses the floor technology of the Big Dachman company for keeping laying hens of the parent herd. It requires significantly less costs for technological equipment and allows the production of higher quality products. The main factor in the production efficiency of a quality hatching egg is the feeding of the parent herd. In the conditions of OJSC Vasilievskaya poultry farm, the cross ROSS 308 is used mainly. Feeding the birds of the parent herd was carried out in accordance with the existing standards using typical full-feed compound feeds prepared at the feed mill according to age and productivity. The cereal part of the feed is represented by wheat, barley and corn. The protein diet is balanced by meal and, according to the age period, its content was $0-20$ days - 20.0; 21-41 - 18; 42-104-14; 105-161 - 14.1515.5; 162-245 - 14.5-15.2 and 246-420 - 14.5-25.5\%\%. To meet energy needs (the exchange energy content in all age periods is $280 \mathrm{MJ} / \mathrm{kg}$ ), sunflower oil is used. Vitamin-mineral supplements in the form of premixes and a drug against mycotoxins (Mycofix Plus) are introduced into the feed.

The chickens were put to experience at the age of 227 days. The experiment was carried out in the workshop for the brood stock of hens. For its holding, part of the workshop was separated by a special temporary partition. Thus, during the experiment, the experimental chickens were in the same conditions of keeping and feeding with the chickens of the main herd, and we can project the results to the entire breeding stock of the workshop. By random sampling, 2 groups were formed (experimental and control) with 100 goals and 14 roosters 
each (table 1). The same table shows the used dosage of DHE introduced into the feed in the form of a premix prepared on the basis of extruded bran.

\section{Results and discussion}

When using DHE, the functional activity of the systems responsible for the antioxidant-antiradical status is significantly and significantly increased. In laying hens, not only the concentration of lipid peroxidation products in the blood decreases, but also the direction of lipid-cholesterol metabolism changes radically. These metabolic changes are crucial in biosynthesis of hens of eggs with a different ratio and a different quality of nutrients.

Moreover, all antioxidants to a greater or lesser degree interrupt the process of peroxidation of cholesterol low density lipoproteins (HLDPL). For this physiological effect, we calculated in the starting working hypotheses of the experiment.

On the 25th day of the experiment, blood was taken from the axillary vein for biochemical studies. From each group of hens, eggs were selected for incubation in the amount of 240 pieces (collection for 3 days). The studied indicators: egg production, preservation, conclusion, hatchability, biochemical parameters of blood and egg yolk.

Along with the analysis of the complex of lipid peroxidation products (MDA), the analysis of cholesterol fractions of lipoproteins of various densities are among the most important diagnostic tests to assess the state of the antioxidant-antiradical system, and are among the most important criteria for nonspecific resistance of the body. Currently, it is believed that indicators such as total lipids, total phospholipids, triacylglycerols and even total cholesterol individually are asymptomatic, i.e., they do not have independent diagnostic value.

When using DHE, as previously shown in broiler chickens (3), the functional activity of the systems responsible for antioxidant-antiradical status is significantly and significantly increased. And first of all, this concerns, with greater or lesser success, the interruption of the lipid peroxidation process, therefore, the decrease in the content of products of their lipid peroxidation MDA including cholesterol of lipoproteins of fractions of different densities (primarily low density lipoprotein cholesterol). In this regard, a determination was made in the blood plasma of chickens: MDA, triacylglycerols (TG); total cholesterol (XO); high density lipoprotein cholesterol (HLPVP); low density lipoprotein cholesterol (HLDPN) and very low density lipoprotein cholesterol (HLPONP). In the yolk of the eggs - MDA.

During the experiment, egg production, hatching qualities of eggs (fertility of hatching eggs, hatching and hatching of chickens) were recorded. After incubation, all other egg defect factors (blood ring, dead, suffocated, weak, fight / cuff) were also taken into account, leading to a decrease in hatch and hatchability. The mass of a whole egg, yolk, protein, and shell was determined. Biochemical parameters were determined using sets of Unimed and Lahema firms.

Despite the sufficient usefulness of the feeding adopted at the poultry farm for chickens of the parent herd, it is necessary to note a high percentage of hatching egg infertility (from 8 to 15\%). Poultry specialists associate this with the quality of the feed used. Poultry diets consisting of compound feed contain a large amount of crude fat, which in the compound feed under normal conditions of storage and feeding is quite easily oxidized. In all likelihood, the administration of DHE to the hens of the experimental group contributed to the normalization of metabolism and changed the ratio of cholesterol lipoprotein fractions.

During the use of DHE in the blood, no significant intergroup differences were found in the concentration of triacylglycerols and in the cholesterol content of the very low density lipoprotein fraction. The concentration of total cholesterol decreased by $8.9 \%$. The cholesterol content in the high- density lipoprotein fraction (anti-atherogenic factor), considered "useful" cholesterol, doubled $(\mathrm{P}<0.01)$, and the cholesterol content in the low-density lipoprotein fraction "atherogenic factor", considered "harmful", sharply decreased twice $(\mathrm{P}<0.01$, table 2). Significant intergroup differences in glutathioperoxidase activity, protein and albumin concentrations were not found in blood plasma and egg yolk. 
Table 1. Experimental design and dose of DHE administered.

\begin{tabular}{|l|c|l|l|}
\hline \multicolumn{1}{|c|}{ Groups } & Number of laying hens & Number of cocks & \multicolumn{1}{c|}{ Feeding } \\
\hline Control & 100 & 14 & Basic diet (OR) \\
\hline Experiment & 100 & 14 & OR + DGE, $100 \mathrm{mg} / \mathrm{kg} \mathrm{of} \mathrm{feed}$ \\
\hline
\end{tabular}

Table 2. Indicators of lipid-cholesterol metabolism in the blood of chickens $(M \pm m, n=5)$.

\begin{tabular}{|l|c|c|c|c|c|}
\hline \multirow{2}{*}{ Groups } & \multirow{2}{*}{ TG } & \multicolumn{3}{|c|}{ The studied indicators } & \multirow{2}{*}{ HLPONP } \\
\cline { 3 - 6 } & $1.23 \pm 0.17$ & HLPVP & XLPVP & HLPNP & $0.56 \pm 0.06$ \\
\hline Control & $1.22 \pm 0.19$ & $5.63 \pm 0.84$ & $2.99 \pm 0.26^{* *}$ & $2.09 \pm 0.21^{* *}$ & $0.55 \pm 0.08$ \\
\hline Experience & 99.2 & 91.1 & 200.7 & 50.6 & 98.2 \\
\hline To control, \% & & &
\end{tabular}

TG- triacylglycerol; HLPVP- total cholesterol XLPVP - cholesterol high-density lipoproteins; HLPNP - cholesterol low-density lipoproteins; HLPONP- cholesterol very low-density lipoproteins

Significant intergroup differences in glutathioperoxidase activity, protein and albumin concentrations were not found in blood plasma and egg yolk.

Table 3. Egg laying of chickens (accounting period 41 days) and the results of incubation.

\begin{tabular}{|l|c|c|c|c|}
\hline \multicolumn{1}{|c|}{ Indicators } & $\begin{array}{c}\text { Control } \\
(\mathrm{n}=100)\end{array}$ & $\begin{array}{c}\text { Experience } \\
(\mathrm{n}=100)\end{array}$ & To control, \% & $\begin{array}{c}\text { Additionally received per } \\
100 \text { goals, }(\% \text { to control })\end{array}$ \\
\hline Egg production & 80.08 & 85.90 & 107.3 & 5.82 \\
\hline Chicks received & 80.0 & 91.7 & 114.6 & 11.7 \\
\hline $\begin{array}{l}\text { The deduction of } \\
\text { chickens }\end{array}$ & 80.0 & 91.7 & 114.6 & 11.7 \\
\hline Unfertilized eggs & 12.7 & 2.5 & 19.68 & 10.2 \\
\hline Fertilized & 87.3 & 97.5 & 116.8 & 19.56 \\
\hline Hatchability chickens, & 69.84 & 89.4 & 128.2 & \\
\hline
\end{tabular}

Table 4. The content of malondialdehyde, mol / 1 .

\begin{tabular}{|l|c|c|}
\hline & \multicolumn{2}{|c|}{ Malonic dialdehyde } \\
\hline \multicolumn{1}{|c|}{ Groups } & In blood plasma & In the yolk \\
\hline Controlled & $8.11 \pm 0.72$ & $60.4 \pm 7.59$ \\
\hline Experienced & $6.65 \pm 0.42 * *$ & $22.6 \pm 2.21 * * *$ \\
\hline To control, $\%$ & 82.0 & 37.41 \\
\hline
\end{tabular}

Despite the absence of noticeable differences in the activity of glutothione peroxidase, the DHE used had a significant difference in the content of HDL and HDL, which play a large role in the formation of the antioxidant status of the experimental bird, which determined the higher productive and reproductive qualities of the experimental bird population (table 3 ).

The egg mass in the control and experimental groups on the 25th and 41st day of the experiment varied between $60-65 \mathrm{~g}$, and no significant intergroup differences were revealed by the weight of the yolk, protein, and shell. The differences were egg laying (table 3). In chickens treated with DHE (experimental group), the number of eggs obtained per head was higher than the control group chickens by $7.3 \%(\mathrm{P}<0.1)$, but the difference in egg production when measured in units, expressed as a percentage, is $5,82 \%$ of the absolute percent of units, that is, an additional 5.82 eggs per day were received per 100 goals. The table shows the results of egg collection on the 25 th day of the experiment. 
Eggs can be qualified as dietary, as a product of functional or baby food, since there were significantly less lipid peroxidation compounds harmful to the human body. Moreover, at the current level of biochemical knowledge it is impossible to judge the dietary value of food products only by their cholesterol content. Firstly, a deficiency in the diet of cholesterol is no less harmful than its excess. Secondly, the bulk of cholesterol does not enter the body with food, but is formed during metabolism. Thirdly, the total cholesterol content is too uninformative. As we repeatedly emphasized, the analysis of lipo-cholesterol metabolism in the body of experimental chickens, confirmed by the analysis of lipoperoxides in the blood and yolk of eggs, unequivocally indicates that the meat of experimental chickens and eggs laid by them can rightfully be considered high-value food products having not only a reduced atherogenic effect on the human body, since they contain less lipoperoxides and cholesterol low density lipoproteins; but they also have pronounced anti-atherogenic properties, which is due to the high content of the high density lipoprotein cholesterol fraction.

It should be emphasized that the terms "atherogenitity" and "antiatherogenitity" are used throughout the world only for brevity. For a long time, everyone has been investing a much broader meaning in them, completely not limited only to cardiovascular pathology. This is far from the only, and perhaps not the main information provided by the analysis of cholesterol of various lipoprotein fractions and the associated dyslipemia, a large list of very serious, systemic metabolic disorders is included here.

Antioxidants exhibit activity that inhibits the oxidation of low density lipoprotein molecules (LDL). The mechanism by which antioxidants inhibit LDL oxidation is unknown. It is assumed that, by reducing the formation of free radicals, they protect the LDL-a-tocopherol complex from oxidation, restore the oxidized LDL-a-tocopherol complex, and / or neutralize metal ions involved in oxidative reactions [7, 10]. It was these physiological effects of dihydroethoxychine that we had in mind when setting up the experiment, counting on improving the performance of the systems responsible for the functional state of the nonspecific resistance of laying hens.

As it was said above, the obtained eggs can be qualified as dietary, as a product of functional or baby food, since they contained significantly less lipid peroxidation compounds harmful to the human body.

At the same time, the eggs must be complete for the generation and generation of offspring and with further good viability, since the products of lipid peroxidation are also harmful to the developing bird embryo.

About $99 \%$ of all egg lipids are in its yolk and they are necessary not only to provide energy for the developing embryo, but it is possible primarily for the formation of cell membranes in it. Egg lipids contain a large percentage of phospholipids with unsaturated fatty acids, which are easily oxidized. In this regard, naturally, in hens of the control group that do not additionally receive an antioxidant drug, the concentration of MDA in the yolk of an egg is 8 times higher than their plasma concentration. Therefore, in the hens of the experimental group, the concentration of MDA was significantly lower in comparison with the control, indicating that the use of DHE provides a higher capacity for antioxidant systems. The concentration of MDA in the yolk of the hens of the experimental group was lower by almost $70.0 \%$ relative to the hens of the control group and exceeded its concentration in the blood by only about 3 times. While in the control group this excess was more than seven times. The data presented indicate, firstly, the high intensity of lipid peroxidation processes in it and, secondly, the high efficiency of the DHE antioxidant used, which tripled the concentration of lipoperoxides in the yolk of eggs. The data presented in table 4 allow us to conclude that the main identified biochemical reason for the lower (by 12.76\%) hatching of chickens from eggs and their hatchability (by 21.88\%) obtained from hens of the control group was an increased intensity of processes lipid peroxidation in the yolk of eggs and in the body of roosters.

The results of the experiment confirmed the correctness of the original working hypothesis. Experimental physiological and biochemical data are confirmed by zootechnical data on the laying of chickens, fertilization of hatching eggs, hatching and hatching of chickens. These indicators were higher in the experimental group than in the control group. At the same time, the concentration of cholesterol in the high-density lipoprotein fraction in the experimental group increased significantly, and the cholesterol content in the low-density lipoprotein fraction also clearly decreased. This confirms the previously argued position that this group of indicators should be considered not only in terms of assessing atherogenicity, but also as related to a large list of serious systemic metabolic disorders and a number of pathologies [9]. In the yolk of eggs from chickens treated with dihydroethoxyquin, there was an almost threefold decrease in the concentration of MDA, which characterizes the presence of lipid peroxidation products, superreactive free radicals, leading to a decrease in nonspecific resistance of the body. The consumption of such eggs in food leads to similar changes in the human body. In this regard, eggs obtained from chickens of the 
experimental group can be qualified as dietary, as a product for children's and functional nutrition, since they contained three times less lipid peroxidation products harmful to the human body. We associate all the identified positive effects with an increase in the nonspecific resistance of the laying hens, characterized by the studied indicators of lipid peroxidation and lipid-cholesterol metabolism. Analysis of the tested antioxidant indicates the high efficiency of their influence both on the physiological and biochemical processes in the body of laying hens, and on their productive indicators.

\section{Conclusion}

In general, the revealed zootechnical effect on the productivity of chickens and the incubation quality of eggs obtained from them, the supplemented data on the activity of the concentration of malondialdehyde, as well as a group of indicators characterizing the specifics of lipid-cholesterol metabolism, allow us to conclude that the used dose of the fed drug is harmless and the biological feasibility of introducing into the production of additives to the diets of laying hens dihydroethoxychine. The totality of the information provided confirms the physiological adequacy for laying hens of the introduction of dihydroethoxychine in the specified dose.

\section{References}

1) Alimohammadi-Saraei, M.H., Chamani, M., Seidavi, A.R., Sadeghi, A.A., Amin-Afshar M. 2018. Effect of green tea and rosemary extracts on performance, organ weights and blood parameters of broilers. Journal of Livestock Science 9: 32-42

2) Bindoli A. 1988. Lipid peroxidation in mitochondria. Free Radic Biol Medicine. 5(4):.247-261. doi: 10.1016/0891-5849(88)90018-4.

3) Buzlama V.S. 1996. The general resistance of animals under stress and its regulation by adaptogens. Reports of the RAAS 1 36-9 for roosters of the parent herd Poultry.8: 23-25.

4) Galochkin V.A. 2001. New horizons for increasing non-specific resistance and animal productivity (Borovsk: VNIIFBiP) p.97

5) Galochkin V.A., Galochkina V. P., Ostrenko K.S. 2009. Development of theoretical foundations and the creation of new generation anti-stress drugs Agricultural Biology. 2: 43-55

6) Galochkina V.A., 2008. The effect of the new antioxidant dihydroethoxychin on non-specific resistance, roductivity and production quality of broiler chickens Problems of Biology of Productive Animals. 2:52-58

7) Lauridsen C., Jakobsen K., Hansen T.K. 1995. The influence of dietary ethoxyquin on the vitamin E status in broilers. Arch Tierernahr. 47 (3):245-254. doi: 10.1080/17450399509381809.

8) Leong V.Y., Brown T.P. 1992. Toxicosis in broiler chicks due to excess dietary ethoxyquin. Avian Dis.36(4): 1102-1106.

9) Middleton T F, Ferket P R, Boyd L C 2001 The effect of ethoxyquin on the quality of ground mitochondrial res J. Toxicol. Environ. Health. 33(2): 229-36

10) Ostrenko K. S., Galochkin V.A., Boryaev G.I., Galochkina V.P. 2018. Role of antioxidants in feed for roosters of the parent herd. Poultry. 2018. 8: 23-25.

11) Reyes J.L., Hernández M.E., Meléndez E., Gómez-Lojero C. 1995. Inhibitory effect of the antioxidant ethoxyquin on electron transport in the mitochondrial respiratory chain. Biochem Pharmacol. 49(3):.283289. doi: 10.1016/0006-2952(94)00487-7.

12) Sethy, K., Swain, P., Behera, K., Sahoo, N., Agrawalla, J., Khadanga S., Mahapatra M.R. and Parhi S.S. 2017. Effect of turmeric (Curcuma longa) supplementation on antioxidants and immunity of broiler birds. Journal of Livestock Science 8: 103-106

13) Travis J.C., Thornton S., Daignault L. 1989. Effect of Santoquin on humoral immune function in mice: lack of interference with selenium utilization. Immunol Cell Biol. 67 (Pt1):83-84. doi: 10.1038/icb.1989.11.

14) Wang S.Y., Bottje W., Maynard P., Dibner J., Shermer W.. 1997. Effect of Santoquin and oxidized fat on liver and intestinal glutathione in broilers. Poult Sci. 76(7): 961-967. doi: 10.1093/ps/76.7.961.

15) Yesuf, K.Y., Mersso, B.T., Bekele T.E. 2017. Effects of different levels of turmeric, fenugreek and black cumin on carcass characteristics of broiler chicken. Journal of Livestock Science 8: 11-17 